\title{
ANÁLISIS DE PROYECTOS DE APRENDIZAJE-SERVICIO DISEÑADOS POR MAESTROS EN FORMACIÓN INICIAL
}

\author{
ANALYSIS OF SERVICE-LEARNING PROJECTS DESIGNED BY PRE- \\ SERVICE PRIMARY TEACHERS
}

\author{
Elena Bravo Lucas, José María Marcos-Merino, Emilio Costillo Borrego, \\ Rocío Esteban Gallego.
}

Universidad de Extremadura

\author{
Correspondencia: José María Marcos-Merino \\ Correo: jmmarcos@unex.es \\ Recibido: 31-03-2020 Aceptado:25/05/2020 \\ DOI: $10.17398 / 0213-9529.40 .1 .5$
}

\section{RESUMEN}

El aprendizaje-servicio es una metodología didáctica que se basa en el aprendizaje de contenidos mediante la realización de un servicio para atender una necesidad social del entorno del alumnado. La investigación educativa apoya su empleo en distintos niveles de enseñanza, entre ellos la formación inicial del profesorado en la que, además de favorecer el aprendizaje conceptual, facilita el desarrollo de competencias relacionadas con la docencia. En este trabajo se analizan 8 proyectos de aprendizaje-servicio diseñados e implementados por futuros maestros. La mayoría de los proyectos están orientados a resolver problemáticas relacionadas con la gestión de residuos y la falta de recursos básicos, planteando objetivos de aprendizaje y de servicio relacionados con la sensibilización hacia estas problemáticas. Dichos proyectos se difunden mediante las redes sociales y la mayoría se implementa parcialmente, debido a la falta de colaboración de las distintas instituciones implicadas.

Palabras clave: Aprendizaje-Servicio; Maestros en formación; Formación inicial del profesorado.

\begin{abstract}
Service-learning is a didactic methodology that pursues content learning by performing a service with which to solve a social need in the students' environment. Educational research supports its use in different educational levels, including initial teacher training in which, in addition to promoting conceptual learning, facilitates the development of competences related to teaching. In this work we analyse 8 service-learning projects designed and implemented by future Primary teachers. Most of these projects are geared towards solving problems related to waste management and lack of basic resources, raising learning and service objectives related to the awareness of these problems. Designed projects were disseminated through social networks and most of them were partially implemented, due to the lack of cooperation of involved organizations.
\end{abstract}

Keyword: Service-Learning, Pre-service Primary teachers, Initial teachers' training. 


\section{MARCO TEÓRICO}

\section{La metodología Aprendizaje-Servicio}

El aprendizaje-servicio (ApS) es una metodología didáctica que se basa en el aprendizaje de conocimientos a la vez que se presta un servicio a la comunidad para atender alguna necesidad presente en la misma. Es una experiencia que combina la acción y la reflexión; donde, a través de una actividad de servicio a la comunidad, el alumnado aplica contenidos, desarrolla competencias y resuelve problemas reales, dentro de un marco académico (Capella, Gil, Martí y Chiva, 2015). Así, esta metodología integra objetivos de servicio con objetivos de aprendizaje, con el objeto de que las actividades desarrolladas generen cambios tanto a quien recibe el servicio como a quien lo da (Aramburuzabala y García, 2012). No se trata de una metodología educativa novedosa, puesto que se compone de experiencias y prácticas que ya eran aplicadas anteriormente de forma independiente en los contextos educativos (Puig y Palos, 2006). La novedad y fortaleza de este método reside en el estrecho, coherente y bien articulado vínculo existente entre el aprendizaje y el servicio a la comunidad. Esta característica singular es la que excluye el voluntariado de las propuestas de ApS, puesto que esta metodología no solo debe suponer una contribución para mejorar la calidad de vida y/o la inclusión social de la población, sino que también debe facilitar un aprendizaje académico en el alumnado participante (Martínez, 2008).

De acuerdo con Puig y Palos (2006), esta metodología, que sirve para la educación formal y no formal, en todas las edades y en los distintos espacios temporales, presenta características deseables, aunque no siempre todas estén presentes en los proyectos ApS: tiene como objetivo llevar a cabo un servicio a la comunidad que permita aprender; desencadena procesos de adquisición de conocimientos y competencias para la vida; supone una pedagogía de la experiencia y la reflexión; requiere una red de alianzas entre las instituciones educativas y las entidades sociales; y provoca efectos en el desarrollo personal, así como cambios positivos en las instituciones educativas y en la comunidad que recibe el servicio.

Para que los proyectos ApS sean una herramienta potente de aprendizaje y de desarrollo de competencias, deben elaborarse partiendo de las necesidades reales del entorno específico cercano al alumnado (Aramburuzabala y García, 2012; Wee, 2017). Solo si esta circunstancia se cumple se podrá contribuir a la mejora del aprendizaje y de la comunidad. Y para cumplir con la misma los proyectos de ApS deben seguir tres etapas principales (Tapia, 2010): i) Diagnóstico inicial y planificación del proyecto, ii) Ejecución y iii) Evaluación y sistematización final.

Como metodología didáctica, los proyectos ApS se encuentran enmarcados dentro del conjunto de las metodologías activas. Estas se caracterizan por ser los propios estudiantes los protagonistas de los procesos de aprendizaje. En la actualidad los estudiantes de los diferentes niveles educativos demandan este tipo de enseñanzas, basadas en metodologías innovadoras, en las que se otorga un mayor protagonismo al alumnado, para favorecer la consecución de los objetivos educativos planteados (Ortega-Tudela, Cámara-Estrella y Diaz-Pareja, 2015). Es en este marco donde se puede plantear la metodología ApS como una alternativa, innovadora y activa, a los métodos tradicionales.

Igualmente, la sociedad actual demanda ciudadanos comprometidos y críticos. El diseño e implementación de proyectos de ApS constituye una estrategia en la formación de una ciudadanía crítica y activa, que interviene e influye de forma directa en la vida comunitaria (Pérez y Ochoa, 2017); implicando procesos de investigación activa, de análisis colectivo y de aplicación directa. Esta circunstancia permite que su aplicación sea posible en todas las etapas educativas, 
desde la Educación Infantil hasta la etapa universitaria (Gil y Martí, 2012), siendo incluso propuesta como estrategia docente por la Conferencia de Rectores de las Universidades Españolas en el contexto de la Responsabilidad Social Universitaria (Álvarez, Martínez, González y Buenestado, 2017; CRUE, 2015).

\section{El Aprendizaje-Servicio en la formación inicial de maestros}

Dentro de la etapa universitaria, la formación inicial de maestros constituye un contexto especialmente idóneo para la implementación de proyectos ApS como metodología didáctica, debido a los efectos positivos que tiene esta herramienta en diferentes aspectos importantes en la formación de futuros docentes. Quizá es por ello, que cada vez se emplea más el ApS como estrategia didáctica en la formación inicial de maestros.

Los proyectos ApS bien planificados y ejecutados son efectivos, en primer lugar, como metodologías de enseñanza-aprendizaje de contenidos con estos alumnos. Se ha observado que la realización de proyectos ApS ayuda a mejorar la comprensión de los conceptos de la asignatura, a relacionar contenidos entre sí y a valorar su empleo en la vida cotidiana (Aramburuzabala y García, 2012; Ibarrola-García y Artuch, 2016 y Folgueiras, Luna y Puig, 2013).

En segundo lugar, la realización de proyectos basados en esta metodología genera efectos positivos en el desarrollo de las competencias y habilidades necesarias para la docencia (Aramburuzabala y García, 2012). Así, estas actividades se caracterizan por su potencial para: i) cambiar percepciones, creencias o prejuicios de los futuros maestros, lo que lo hace ser un elemento de "auténtica mejora de la formación docente" (Opazo, 2015), y ii) desarrollar competencias necesarias para llevar a cabo una enseñanza de calidad (Root, Callagan, y Sepansky, 2002). Dentro de estas competencias está la valoración positiva de la inclusión: la realización de proyectos ApS provoca que el profesorado en formación se vuelva más inclusivo y tenga un mayor interés hacia la diversidad (Corbatón, Moliner, Martí, Gil, y Chiva, 2015; Root, 2005; Vázquez, Liesa, y Lozano, 2017), lo que podría tener efectos positivos en su futura acción docente y en la comunidad en la que esta se desarrolle. También está la capacidad de adaptar el currículum a las demandas de los alumnos y a las necesidades del entorno de estos, como ha observado Root (2005) en futuros docentes participantes en proyectos ApS; así como la mejora de competencias de planificación y comunicación con los padres y representantes de los alumnos (Anderson, 2000). Opazo (2015) añade otros efectos positivos del ApS en la formación inicial de maestros como el desarrollo de una visión más realista de la profesión, una mayor sensibilidad y tolerancia hacia las necesidades de su alumnado, un mejor entendimiento social y emocional del mismo, un mayor desarrollo en la capacidad de liderazgo, una mayor comunicación entre docentes y una reflexión crítica acera de sus propios pensamientos y prácticas.

En tercer lugar, la metodología basada en ApS influye positivamente en las competencias personales y pro-sociales de los maestros en formación, como se ha observado cuando estos desarrollan esta metodología durante sus asignaturas de Prácticum (Blanch, París y Comes, 2017). La implementación de proyectos ApS permite al futuro maestro involucrarse y comprometerse con su comunidad y, por ende, implicarse en las necesidades de la misma de manera reflexiva y crítica. Así, dentro del plano metacognitivo, permite pensar en la educación como una actividad ética, lo que fomenta una mejora de la visión que tienen los futuros maestros sobre la profesión docente (Aramburuzabala, García-Peinado y Elvias, 2013; Coladarci, 1992; Donahue, 1999; García y Cotrina, 2015). 
Los propios maestros en formación reconocen los beneficios de realizar actividades basadas en ApS. Así, entre otros aspectos, destacan que la utilización de esta metodología favorece un aprendizaje significativo y vivencial de los contenidos, aumenta tanto su motivación como docentes como la motivación de sus futuros alumnos, mejora su autoestima y su autoeficacia docente, amplía su conocimiento de su comunidad y de las necesidades sociales de la misma, resulta una fuente de autoconocimiento y visibiliza la relación Universidad-Sociedad (Anderson, 2000; Ayuste, Gros, Payá y Rubio, 2017).

Sin embargo, es necesario conocer las limitaciones de estos proyectos, sobre todo cuando presentan deficiencias en su planificación y ejecución (Wee, 2017). De este modo, no todos los proyectos ApS consiguen mejorar el aprendizaje de los futuros maestros participantes. SantosPastor, Cañadas y Martínez-Muñoz (2020) destacan como limitaciones y dificultades de la metodología ApS en la formación inicial de profesionales la falta de formación y experiencia en esta metodología del alumnado universitario en lo referente a su diseño, intervención y evaluación, la elevada carga de trabajo tanto para el profesorado como para el alumnado y la dificultad para desarrollar acciones coordinadas entre programas, profesorado y alumnado.

En el presente trabajo, se describen ocho proyectos ApS, diseñados e implementados por maestros en formación inicial de la Universidad de Extremadura (estudiantes del último curso del Grado en Educación Primaria, Facultad de Educación), analizando los principales aspectos de los mismos y discutiendo sus implicaciones en los procesos de formación inicial del profesorado.

\section{METODOLOGÍA}

\section{Muestra y contexto}

Para la realización de esta investigación se ha utilizado un muestreo intencional o de conveniencia. La muestra empleada está constituida por 8 proyectos diseñados por 67 maestros en formación inicial (77,6 \% mujeres, 21 años de edad de media), son estudiantes del Grado en Educación Primaria en la Facultad de Educación de Badajoz (Universidad de Extremadura, España), que cursaron la asignatura "Conocimiento del medio natural en Educación Primaria" de cuarto curso durante el año académico 2018-2019.

En el contexto de esta asignatura, los participantes recibieron una clase expositiva en la que se expuso el ApS como metodología didáctica para la enseñanza interdisciplinar y global. En dicha sesión se explicó, entre otros aspectos, en qué consiste el ApS como metodología didáctica, sus principales potencialidades y beneficios, los pasos necesarios para diseñar e implementar proyectos ApS... Asimismo, se mostraron distintos ejemplos de proyectos ApS realizados con alumnado de distintos niveles educativos.

Tras esto, se solicitó a los alumnos un trabajo grupal, en el que debían desarrollar un proyecto ApS basándose en una necesidad social de su entorno. De este modo, los alumnos participantes se organizaron en 8 grupos de trabajo (formados por entre 8 y 9 alumnos), cada uno de los cuales debía diseñar, elaborar, implementar y difundir su propio proyecto ApS. El tiempo medio empleado por los distintos grupos en el desarrollo del proyecto ApS fue aproximadamente de unas 4 semanas.

Cada grupo entregó una memoria de su proyecto ApS en la que se recogía los siguientes apartados: Título, datos técnicos (centros y entidades implicadas, personas responsables del proyecto y personas destinatarias del proyecto), antecedentes, necesidad social que atiende el proyecto, objetivos de servicio, objetivos de aprendizaje, materias y áreas curriculares implicadas, actividades a realizar para alcanzar los objetivos de servicio y de aprendizaje (dentro 
del aula y fuera del aula), difusión del proyecto, recursos humanos y materiales, presupuesto, calendario de aplicación, evaluación del proyecto y reflexión.

\section{Análisis de datos}

Las propuestas de proyectos ApS han sido analizadas siguiendo una metodología de análisis cualitativo. Las categorías que se han establecido para el análisis cualitativo son:

1. Necesidad social que atiende el proyecto ApS.

2. Objetivos de servicio del proyecto ApS.

3. Objetivos de aprendizaje del proyecto ApS.

4. Actividades diseñadas para alcanzar los objetivos planteados y atender la necesidad social que fundamenta el proyecto ApS.

5. Grado de implementación del proyecto ApS.

6. Mecanismos de difusión del proyecto ApS.

Este proceso de análisis cualitativo ha sido realizado por 4 investigadores en Didáctica de las Ciencias.

\section{RESULTADOS Y DISCUSIÓN}

\section{Análisis de las experiencias}

El análisis de las ocho propuestas de experiencias de ApS diseñadas e implementadas por maestros en formación inicial de la Universidad de Extremadura (Tablas 1 a 4), permite agruparlas en 4 categorías según la temática principal de los proyectos:

1. Gestión de residuos y reciclaje (Tablas 1 y 2): Proyectos "Si yo puedo, jtú también!", "Tu papel es importante", "Apostando por un mercadillo limpio" y "Tu basura es la de todos".

2. Falta de recursos básicos (Tabla 3): Proyectos "Ponte en su lugar" y "Recogida solidaria".

3. Consumismo (Tabla 4): Proyecto "Black Friday".

4. Especies invasoras (Tabla 4): Proyecto "Origen, evolución y concienciación sobre el camalote en el río Guadiana".

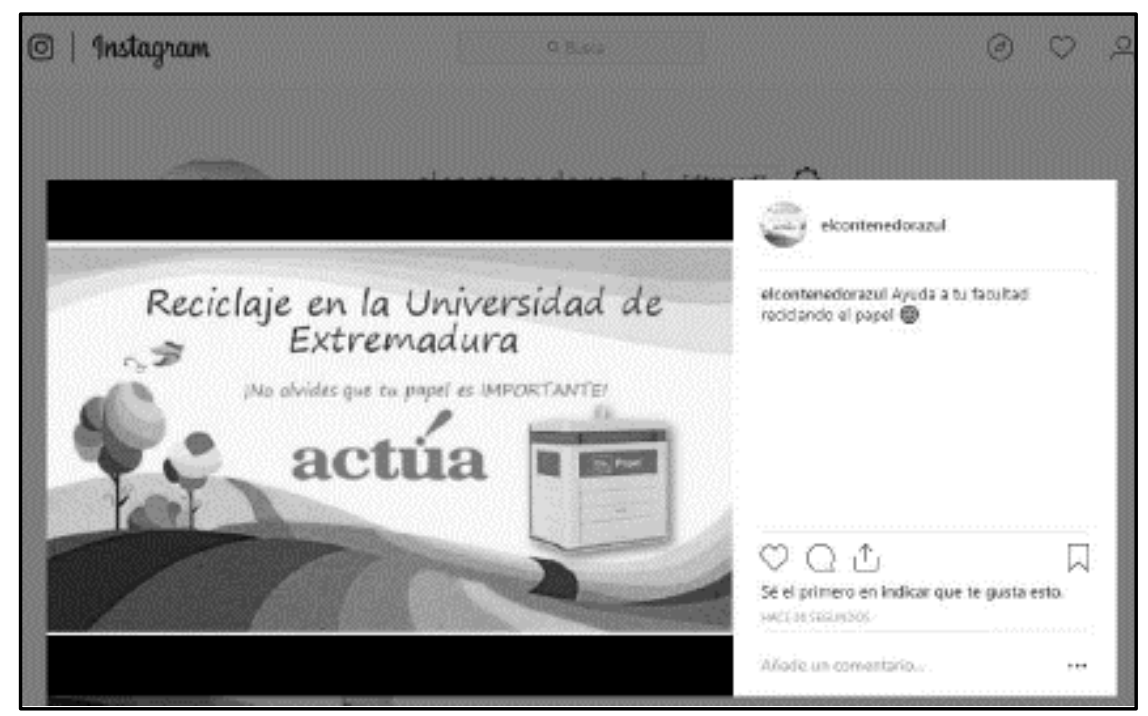

Figura 1. Ejemplo de divulgación del proyecto ApS "Tu papel es importante". 
Tabla 1. Análisis de los dos proyectos ApS sobre gestión de residuos y reciclaje "Si yo puedo, itú también!" y "Tu papel es importante"

\section{Gestión de residuos y reciclaje 1}

\begin{tabular}{|c|c|c|}
\hline Título & Si yo puedo, ¡tú también! & Tu papel es importante \\
\hline $\begin{array}{l}\text { Necesidad } \\
\text { social }\end{array}$ & $\begin{array}{l}\text { Disminuir la cantidad de los residuos } \\
\text { (vidrio y plásticos) que quedan en el } \\
\text { suelo del recinto habilitado para } \\
\text { hacer botellones en la ciudad } \\
\text { Badajoz, debido a falta de } \\
\text { contenedores específicos y de } \\
\text { concienciación de los asistentes. }\end{array}$ & $\begin{array}{l}\text { Promover el reciclaje de papel en la } \\
\text { Facultad de Educación de la Universidad } \\
\text { Extremadura, ya que no existen } \\
\text { contendores para dicho fin y los } \\
\text { estudiantes y trabajadores se deshacen } \\
\text { de él en contenedor general. }\end{array}$ \\
\hline $\begin{array}{l}\text { Objetivos de } \\
\text { servicio }\end{array}$ & $\begin{array}{l}\text { Conseguir que el Ayuntamiento } \\
\text { aumente las papeleras y/o } \\
\text { contenedores específicos en la } \\
\text { ubicación de los botellones. } \\
\text { Concienciar a los asistentes al } \\
\text { botellón con relación a la gestión } \\
\text { correcta de sus residuos. }\end{array}$ & $\begin{array}{l}\text { Aumentar la cantidad de papel reciclado } \\
\text { por los usuarios de la Facultad de } \\
\text { Educación. } \\
\text { Concienciar a los responsables de la } \\
\text { Facultad de Educación de la necesidad de } \\
\text { contenedores específicos para reciclar el } \\
\text { papel usado por los estudiantes y } \\
\text { trabajadores del centro. }\end{array}$ \\
\hline $\begin{array}{l}\text { Objetivos de } \\
\text { aprendizaje }\end{array}$ & $\begin{array}{l}\text { Conocer e implantar las } \\
\text { metodologías ApS y aprendizaje } \\
\text { cooperativo. } \\
\text { Aprender sobre el reciclaje. } \\
\text { Asumir responsabilidades para } \\
\text { lograr el desarrollo sostenible. }\end{array}$ & Ninguno \\
\hline Actividades & $\begin{array}{l}\text { Contacto con responsables } \\
\text { Ayuntamiento para solicitar un } \\
\text { aumento de contenedores y/o } \\
\text { papeleras. } \\
\text { Elaboración y divulgación de } \\
\text { carteles de concienciación sobre la } \\
\text { importancia de no dejar los residuos } \\
\text { en el suelo y del reciclaje. }\end{array}$ & $\begin{array}{l}\text { Comunicación con responsables de la } \\
\text { Facultad para solicitar la colocación de } \\
\text { contenedores de papel. } \\
\text { Creación de cuentas de concienciación en } \\
\text { redes sociales (tituladas "El contenedor } \\
\text { azul"). } \\
\text { Elaboración de carteles de concienciación } \\
\text { sobre el reciclado de papel. }\end{array}$ \\
\hline $\begin{array}{l}\text { Grado de } \\
\text { implementación. } \\
\text { Difusión. }\end{array}$ & $\begin{array}{l}\text { Divulgación de carteles en las redes } \\
\text { sociales de los alumnos del grupo } \\
\text { para concienciar sobre los residuos } \\
\text { que dejan en el suelo. } \\
\text { Respuesta negativa de } \\
\text { responsables del Ayuntamiento } \\
\text { sobre colocar más contenedores en } \\
\text { la zona de botellón. }\end{array}$ & $\begin{array}{l}\text { Divulgación de carteles en las redes } \\
\text { sociales "El contenedor azul" y en las } \\
\text { propias de los alumnos del grupo (Figura } \\
\text { 1) para concienciar sobre el reciclaje del } \\
\text { papel que usan. } \\
\text { Respuesta negativa de responsables del } \\
\text { centro sobre la colocación de un } \\
\text { contenedor de papel. }\end{array}$ \\
\hline
\end{tabular}

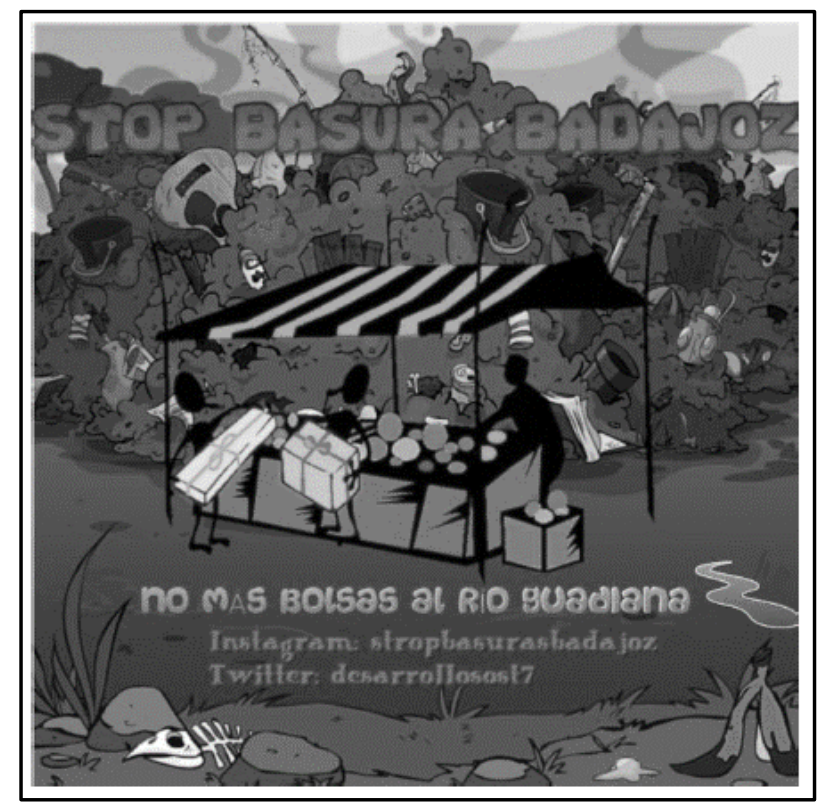

Figura 2. Ejemplo de divulgación del proyecto ApS "Apostando por un mercadillo limpio". 
Tabla 2. Análisis de dos proyectos ApS sobre gestión de residuos y reciclaje "Apostando por un mercadillo limpio" $y$ "Tu basura es la de todos".

\begin{tabular}{|c|c|c|}
\hline \multicolumn{3}{|c|}{ Gestión de residuos y reciclaje 2} \\
\hline Título & Apostando por un mercadillo lim & $\begin{array}{l}\text { Tu basura es la de } \\
\text { todos }\end{array}$ \\
\hline $\begin{array}{l}\text { Necesidad } \\
\text { social }\end{array}$ & $\begin{array}{l}\text { Disminuir la cantidad de residuos, } \\
\text { (fundamentalmente plásticos y } \\
\text { cartones) que quedan en el lugar donde } \\
\text { se celebra el mercadillo en la ciudad de } \\
\text { Badajoz, debido a la falta de } \\
\text { contenedores específicos y/o papeleras } \\
\text { y de concienciación de comerciantes y } \\
\text { asistentes. }\end{array}$ & $\begin{array}{l}\text { Reducir la gran cantidad de residuos } \\
\text { que hay en el suelo de las zonas de } \\
\text { paseo cercanas al río Guadiana. }\end{array}$ \\
\hline $\begin{array}{l}\text { Objetivos de } \\
\text { servicio }\end{array}$ & $\begin{array}{l}\text { Concienciar a los comerciantes y } \\
\text { asistentes al mercadillo sobre la } \\
\text { recogida de residuos y el reciclaje. } \\
\text { Conseguir que el Ayuntamiento } \\
\text { aumente las papeleras y/o } \\
\text { contenedores específicos en la } \\
\text { ubicación del mercadillo. }\end{array}$ & $\begin{array}{l}\text { Concienciar a la sociedad acerca de } \\
\text { las consecuencias que producen los } \\
\text { residuos en el medio ambiente y } \\
\text { fomentar el respeto y los buenos } \\
\text { hábitos hacia el medio ambiente. }\end{array}$ \\
\hline
\end{tabular}

Conocer el impacto ambiental de los Valorar la importancia del desarrollo residuos plásticos, principalmente en sostenible en las comunidades.

Objetivos de ecosistemas como el río.

aprendizaje

Desarrollar conciencia sobre el uso de plásticos y sus consecuencias para el desarrollo sostenible.
Fomentar el desarrollo de hábitos responsables de consumo.

Conocer las consecuencias de la contaminación.
Visita al mercadillo para conocer el grado de generación de residuos y las medidas de recogida existentes.

Solicitar al Ayuntamiento un aumento en las medidas de recogida y la instalación de un mayor número de contenedores.

Actividades Contacto con cadenas de radio para crear una campaña de concienciación ciudadana.

Creación de cuentas en las redes sociales para publicar mensajes de concienciación.

Realizar una campaña de sensibilización en el mercadillo.
Contactar con el Ayuntamiento para organizar una marcha de concienciación y recogida de residuos.

Divulgación de la marcha en el aula de $4^{\circ}$ EP en la Facultad de Educación y a través de redes sociales. Realización de la marcha para la recogida de residuos en las zonas de paseo cerca del río Guadiana. Autoevaluación de los participantes.

\begin{tabular}{|c|c|}
\hline $\begin{array}{l}\text { Grado de } \\
\text { implementación. } \\
\text { Difusión. }\end{array}$ & $\begin{array}{l}\text { Divulgación de la campaña de } \\
\text { sensibilización en redes sociales y } \\
\text { mediante carteles y charlas en el } \\
\text { mercadillo (Figura 2). } \\
\text { Respuesta negativa de los responsables } \\
\text { del Ayuntamiento y de los de las } \\
\text { cadenas de radio ante la propuesta. }\end{array}$ \\
\hline
\end{tabular}

Respuesta positiva del Ayuntamiento e integración de la marcha propuesta con otra marcha organizada desde el Ayuntamiento.

Realización de la marcha en colaboración con el Ayuntamiento y empresas patrocinadoras.

Difusión del proyecto implementado a través de Facebook, páginas webs y prensa local (Figura 3). 


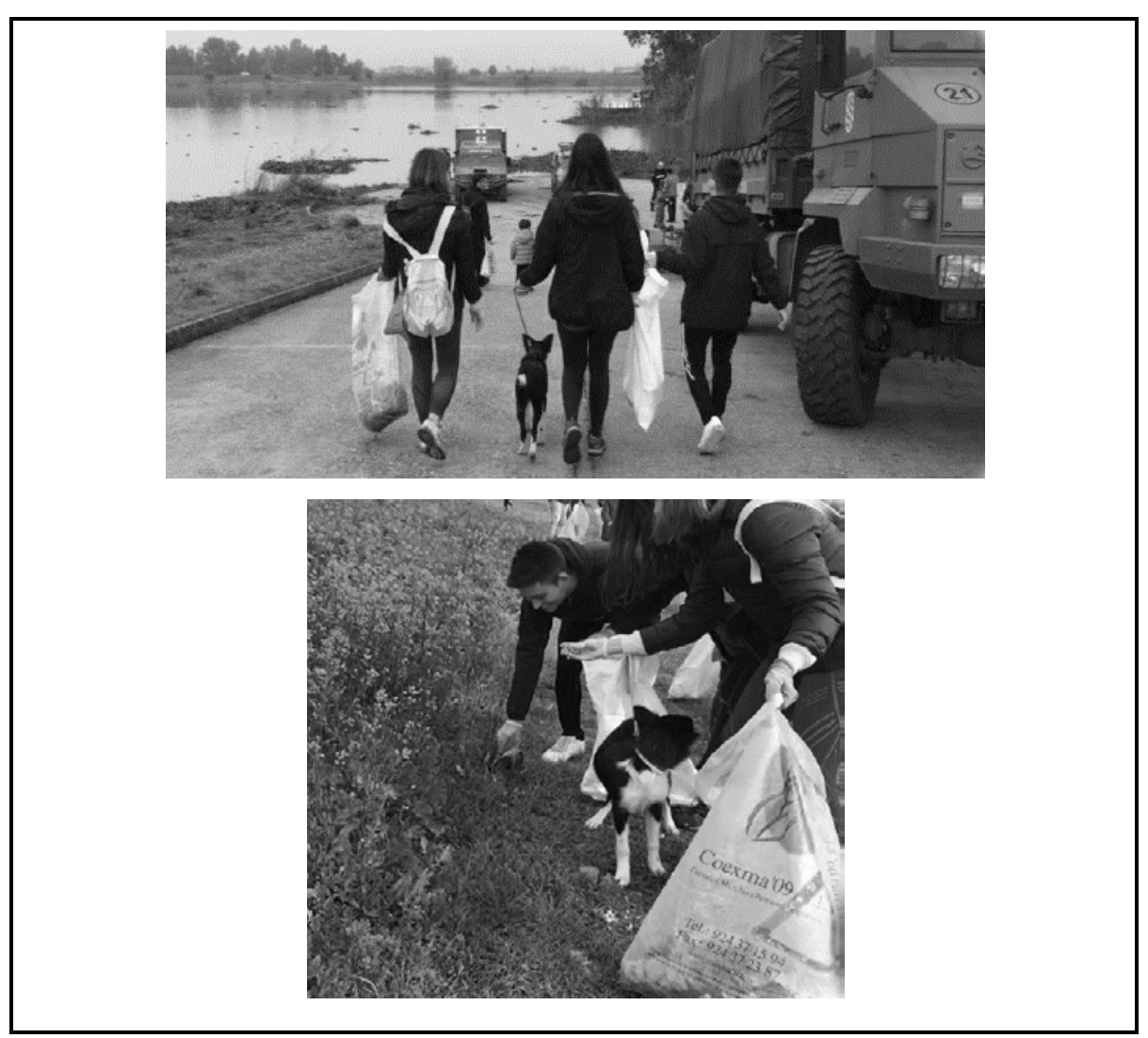

Figura 3. Fotografías de futuros maestros participantes en el proyecto "Tu basura es la de todos" durante la marcha para la recogida de residuos en el paseo del río Guadiana.

Tabla 3. Análisis de los proyectos sobre la falta de recursos básicos "iPonte en su lugar!" y "Recogida solidaria".

\begin{tabular}{|c|c|c|}
\hline \multicolumn{3}{|c|}{ Falta de recursos básicos } \\
\hline Título & ¡Ponte en su lugar! & Recogida solidaria \\
\hline $\begin{array}{l}\text { Necesidad } \\
\text { social }\end{array}$ & $\begin{array}{l}\text { Falta de conciencia sobre la cantidad de } \\
\text { personas de la ciudad Badajoz que, por } \\
\text { falta de recursos, necesitan acudir al } \\
\text { comedor social de Badajoz. }\end{array}$ & $\begin{array}{l}\text { Falta de conciencia sobre la cantidad } \\
\text { de familias de la ciudad Badajoz que } \\
\text { por su situación precaria o de falta de } \\
\text { recursos acuden a Cáritas. }\end{array}$ \\
\hline $\begin{array}{l}\text { Objetivos } \\
\text { de servicio }\end{array}$ & $\begin{array}{l}\text { Sensibilizar a los ciudadanos de } \\
\text { Badajoz sobre la cantidad de personas } \\
\text { que no tienen cubiertas las necesidades } \\
\text { básicas y sobre la necesidad de } \\
\text { colaborar con el comedor social de la } \\
\text { ciudad. }\end{array}$ & $\begin{array}{l}\text { Recoger ropa en buen estado. } \\
\text { Concienciar a la población sobre la } \\
\text { situación de muchas familias. } \\
\text { Fomentar valores como la empatía y } \\
\text { la solidaridad. } \\
\text { Dar respuesta a las necesidades de } \\
\text { las Familias. }\end{array}$ \\
\hline $\begin{array}{l}\text { Objetivos } \\
\text { de aprendizaje }\end{array}$ & $\begin{array}{l}\text { esarrollar los objetivos de desarrollo } \\
\text { stenible, especialmente el Objetivo } 2 \text { : } \\
\text { ambre cero. }\end{array}$ & $\begin{array}{l}\text { Conocer y valorar los objetivos de } \\
\text { desarrollo sostenible. }\end{array}$ \\
\hline Actividades & $\begin{array}{l}\text { Búsqueda de información. } \\
\text { Realización de una campaña de } \\
\text { concienciación. } \\
\text { Recoger alimentos en la Facultad de } \\
\text { Educación para llevarlos al comedor } \\
\text { social. }\end{array}$ & $\begin{array}{l}\text { Contactar con Cáritas para conocer } \\
\text { las necesidades específicas. } \\
\text { Difundir el proyecto en la Facultad de } \\
\text { Educación. } \\
\text { Sensibilizar a los estudiantes } \\
\text { mediante fotografías. } \\
\text { Recoger ropa en la Facultad } \\
\text { Educación para entregarla a la } \\
\text { Parroquia de San Fernando. }\end{array}$ \\
\hline $\begin{array}{l}\text { Grado de } \\
\text { implementación. } \\
\text { Difusión. }\end{array}$ & $\begin{array}{l}\text { El centro no autorizó colocar } \\
\text { contenedores de recogida de alimentos } \\
\text { en el hall de la Facultad, pero sí en el } \\
\text { aula de la asignatura, para llevarlos al } \\
\text { comedor social de Badajoz. } \\
\text { Creación de un perfil de Facebook } \\
\text { donde difundir la información del } \\
\text { proyecto. }\end{array}$ & $\begin{array}{l}\text { El centro no autorizó colocar } \\
\text { contenedores de recogida de ropa en } \\
\text { el hall de la Facultad, pero sí en el } \\
\text { aula de la asignatura, para llevarlos } \\
\text { Cáritas. } \\
\text { Colocación de carteles en la Facultad } \\
\text { de Educación (Figura 4). }\end{array}$ \\
\hline
\end{tabular}

Campo Abierto, v. 40, n. 1, p. 5-19, 2021 


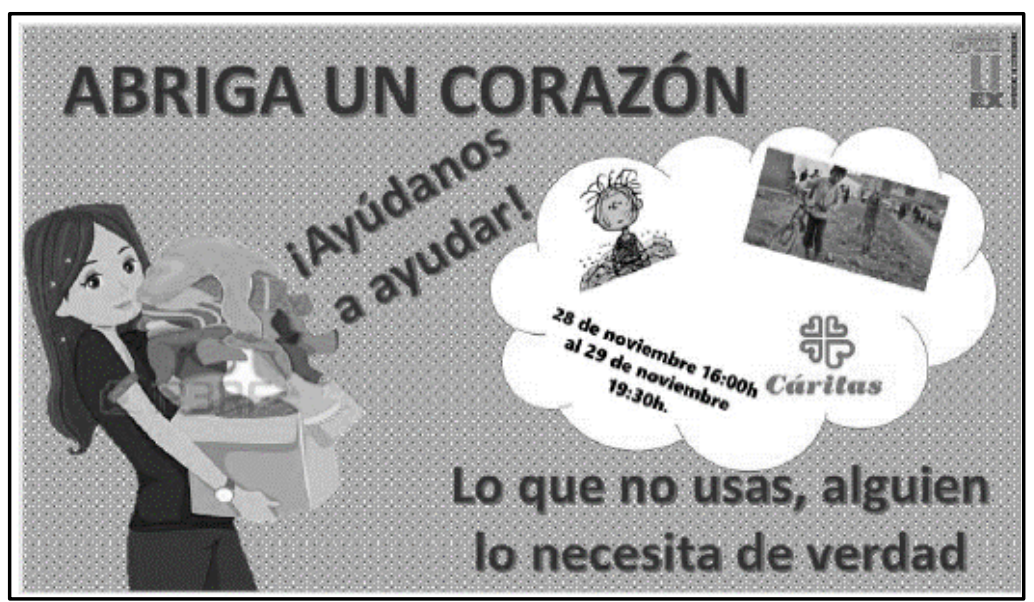

Figura 4. Ejemplo de divulgación del proyecto ApS "Recogida solidaria".

Tabla 4. Análisis de los proyectos sobre consumismo "Black Friday" y sobre especies invasoras "Origen, evolución y concienciación sobre el camalote en el río Guadiana."

\begin{tabular}{|c|c|c|}
\hline \multicolumn{3}{|c|}{ Consumismo y especies invasoras } \\
\hline Título & Black Friday & $\begin{array}{c}\text { Origen, evolución y concienciación } \\
\text { sobre el camalote en el río } \\
\text { Guadiana. }\end{array}$ \\
\hline $\begin{array}{l}\text { Necesidad } \\
\text { social }\end{array}$ & $\begin{array}{l}\text { Frenar el consumismo y la compra } \\
\text { compulsiva de productos innecesarios. }\end{array}$ & $\begin{array}{l}\text { Preservar el ecosistema de los ríos de } \\
\text { Badajoz. }\end{array}$ \\
\hline $\begin{array}{l}\text { Objetivos de } \\
\text { servicio }\end{array}$ & $\begin{array}{l}\text { Concienciar a alumnos de ESO sobre } \\
\text { el consumo responsable. }\end{array}$ & $\begin{array}{l}\text { Participar en la retirada de camalote } \\
\text { junto a la UME. } \\
\text { Concienciar sobre el daño que } \\
\text { producen las especies invasoras a } \\
\text { alumnos de la Facultad Educación. }\end{array}$ \\
\hline $\begin{array}{l}\text { Objetivos de } \\
\text { aprendizaje }\end{array}$ & $\begin{array}{l}\text { Conocer e implantar metodologías de } \\
\text { trabajo cooperativo. } \\
\text { Adquirir conciencia sobre el } \\
\text { consumismo y sus consecuencias para } \\
\text { el desarrollo sostenible. } \\
\text { Desarrollar hábitos de consumo } \\
\text { responsable. }\end{array}$ & $\begin{array}{l}\text { Conocer el daño que causa el camalote } \\
\text { en el río. } \\
\text { Aprender las características más } \\
\text { relevantes del camalote. }\end{array}$ \\
\hline Actividades & $\begin{array}{l}\text { Contacto con responsables del centro } \\
\text { comercial El Faro de Badajoz para } \\
\text { grabar un vídeo de concienciación. } \\
\text { Grabación de un vídeo sobre el } \\
\text { consumismo, las ofertas engañosas y } \\
\text { el Black Friday. } \\
\text { Elaboración de carteles de } \\
\text { concienciación sobre el consumismo. } \\
\text { Contacto con docentes de ESO para } \\
\text { ofrecer charlas sobre consumo } \\
\text { responsable y desarrollo sostenible. }\end{array}$ & $\begin{array}{l}\text { Colaboración en la retirada del } \\
\text { camalote. } \\
\text { Búsqueda de información, video } \\
\text { informativo, noticias relacionadas para } \\
\text { preparar charlas-debates de } \\
\text { concienciación. } \\
\text { Salida al parque. } \\
\text { Realización de carteles informativos. }\end{array}$ \\
\hline $\begin{array}{l}\text { Grado de } \\
\text { implementación. } \\
\text { Difusión. }\end{array}$ & $\begin{array}{l}\text { Respuesta negativa del responsable } \\
\text { del centro comercial, por lo que el vídeo } \\
\text { se elaboró en la calle Menacho puesto } \\
\text { que en ella hay un alto número de } \\
\text { tiendas. } \\
\text { Divulgación de los carteles y del vídeo } \\
\text { en redes sociales de los alumnos del } \\
\text { grupo. } \\
\text { Charlas a alumnos de ESO. }\end{array}$ & $\begin{array}{l}\text { Respuesta negativa por parte de los } \\
\text { responsables para colaborar en la } \\
\text { recogida. } \\
\text { Realización de un cartel que no se } \\
\text { llegó a difundir. }\end{array}$ \\
\hline
\end{tabular}




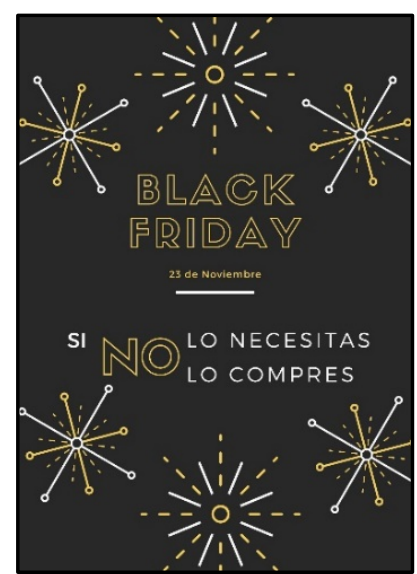

Figura 5. Ejemplo de divulgación del proyecto ApS "Black Friday".

El análisis global de los 8 proyectos ApS elaborados por los futuros maestros indica que:

- La mitad de las propuestas ApS diseñadas (50\%) están destinadas a solucionar necesidades sociales relacionadas con la gestión de los residuos y el reciclaje, un $25 \%$ está orientado a solventar la falta de diferentes recursos básicos en población en riesgo de exclusión social y el $25 \%$ restante aborda otros problemas sociales como el consumismo y las especies invasoras.

- $\quad$ Todas las propuestas presentadas por los participantes atienden necesidades sociales cercanas al entorno del alumnado, llevándose todas a cabo en la ciudad de Badajoz. Una de ellas se centra en una necesidad exclusiva de la Facultad Educación y el resto en problemas de la ciudad.

- $\quad$ Todos los proyectos ApS presentados recogen la concienciación y/o sensibilización de la población en relación a las distintas temáticas trabajadas como uno de los objetivos de servicio planteados, y todos, excepto uno (12\% del total) alcanzan este objetivo. El $36 \%$ de los proyectos (3 propuestas) también plantea como objetivo de servicio que la institución correspondiente se implique en la solución del problema, aunque ninguno de ellos consigue una respuesta positiva de la misma. Y el $64 \%$ restante (5 propuestas) propone, como objetivo de servicio, conseguir autorización de las instituciones correspondientes para llevar a cabo acciones destinadas a solucionar las problemáticas: Dos instituciones no autorizan dichas acciones y tres la autorizan con ciertas modificaciones.

- Las formas de alcanzar el objetivo de concienciación de la ciudadanía más repetidas son la elaboración de carteles y/o videos con campañas de concienciación (empleado en el 75 $\%$ de las propuestas) y la difusión de los mismos a través de redes sociales como Twitter, Facebook o Instagram, bien de los propios alumnos o bien creando cuentas específicas para los proyectos diseñados (en el $75 \%$ de los casos).

- Respecto a los objetivos de aprendizaje, la mayoría de las propuestas se centran en conocer las diferentes problemáticas abordadas y en fomentar la concienciación y la contribución a los objetivos de desarrollo sostenible. Uno de los proyectos planteados (12, $5 \%$ del total) no define ningún objetivo de aprendizaje.

- $\quad$ Respecto al grado de implementación de los proyectos ApS diseñados, dos de las ocho propuestas diseñadas por los futuros maestros se implementan en su totalidad ( $25 \%$ de los proyectos ApS): "Ponte en su lugar" (se recogen alimentos en la Facultad Educación, previa divulgación del proyecto, y se llevan al comedor de Badajoz) y "Origen, evolución y concienciación sobre el camalote en el río Guadiana" (se lleva a cabo la marcha de concienciación y recogida de basura en el paseo del río Guadiana en colaboración con el Ayuntamiento y se difunde el proyecto ApS implementado). Los seis proyectos ApS restantes ( $75 \%$ de las propuestas) no se pueden implementar completamente debido a la falta de implicación de los distintos centros y responsables, tanto públicos (Facultad Educación y Ayuntamiento de Badajoz) como privados (centro comercial El Faro), implicados en las actividades planteadas dentro de los mismos. Sin embargo, la parte del proyecto que depende únicamente del alumnado que propone el proyecto sí se desarrolla por completo. Por último, uno de los proyectos ("Black Friday") se modifica con el fin de poder implementarlo sin la colaboración del centro comercial. 


\section{DISCUSIÓN}

La mayoría de las propuestas de proyectos ApS realizadas por los maestros en formación inicial de la Facultad Educación estaban enfocadas a problemas de su entorno relativos a la gestión de residuos y a la falta de recursos básicos. Sin embargo, ninguno de los proyectos presentados está centrado en mejorar la atención educativa de algún colectivo del entorno de los futuros maestros. Solo el proyecto "Black Friday" tenía como uno de sus objetivos informar a alumnos de ESO sobre consumo responsable y desarrollo sostenible. Este resultado no concuerda con algunas investigaciones previas (Aramburuzabala y García, 2012; Martín y Rascón, 2015; Opazo, 2015; Rodríguez y Gómez Escobar, 2019); según las cuales los futuros maestros, debido al Grado que están cursando y a su vocación profesional, suelen proponer experiencias ApS relacionadas con fines educativos. Una posible causa, que podría explicar las temáticas elegidas para los proyectos ApS, podría ser la propia naturaleza de la asignatura en la que se enmarca la realización de estas propuestas, una asignatura de didáctica de las ciencias ("Conocimiento del medio natural en Educación Primaria"), en la que se imparten, entre otros, contenidos relacionados con el desarrollo sostenible, la preservación del medio ambiente y la gestión de los recursos. Otro posible motivo por el cual los futuros maestros participantes en este estudio han elegido temáticas alejadas del ámbito educativo podría estar relacionado con sus propias motivaciones e intereses personales (American Association of Higher Education, 2002).

Los proyectos ApS presentados en este estudio muestran, asimismo, un equilibrio entre los objetivos de servicio y de aprendizaje propuestos por los futuros maestros, lo que coincide con la investigación desarrollada por Sigmon (1997). Según este autor, este hecho aporta estabilidad a un proyecto ApS. Además, se observa que muchos de estos objetivos y de las necesidades detectadas están relacionadas con el medio natural, lo que está en consonancia con la temática de la asignatura dentro de la cual se han elaborado. Aunque en ningún caso los objetivos de aprendizaje profundizan en el conocimiento del contenido del currículo o en los contenidos científicos relacionados con las problemáticas abordadas. La concordancia entre objetivos del servicio y objetivos de aprendizaje, siempre que estos estén planteados al nivel que se trabaja en la asignatura, favorece el aprendizaje de la materia, según lo plantado por Ramírez, Aramburuzabala y Cerrillo (2014); quienes afirman que los programas de ApS deben estar relacionados con los objetivos del plan de estudios en el que se desarrollen las propuestas, porque esto proporciona una mayor riqueza en el aprendizaje de la asignatura.

Las necesidades detectadas por los maestros en formación inicial participantes están relacionadas con su entorno, son problemas de su ciudad muy cercanos a ellos: los residuos que quedan en el suelo de la zona donde realizan el botellón o de la zona del río por la cual pasean, el reciclaje en su propio centro de estudios, la recogida de alimentos y ropa en favor de un comedor social y parroquia cercanos o la preservación del ecosistema del río mediante la retirada de especies invasoras del mismo. Esta observación coincide con varios estudios previos (Briede y Mora, 2016; Rodríguez, de la Herrán y Cortina, 2015; Francisco y Moliner, 2010) que han mostrado que la realización de proyectos ApS despierta en los futuros maestros un compromiso con su entorno más cercano; lo que fomenta una mayor conciencia en cuanto a responsabilidad, sensibilidad y acción solidaria, vinculando la Universidad con las necesidades sociales.

Respecto a los mecanismos empelados para divulgar a priori o difundir a posteriori los proyectos diseñados, el uso de diferentes redes sociales es el mecanismo empleado en la mayoría de los proyectos ApS de este estudio. Esta circunstancia se ha observado previamente en otras experiencias de ApS (Gillanders, Cores y Tojeiro, 2018; Priede, López-Cózar, RuizMorales y Baena, 2019; Tello, Cerrillo, Mateos y Aramburuzabala, 2017), en las que se ha 
destacado el alto poder de difusión y movilización ciudadana de estas herramientas como vías para mejorar la implementación de los proyectos ApS y para difundir los resultados de los mismos.

Finalmente, y con relación a la implementación de los proyectos diseñados, el 75\% de estos queda limitado al desarrollo del servicio social realizado íntegramente por los alumnos, debido a la falta de colaboración de las entidades implicadas. Este hecho, de acuerdo con el análisis de las reflexiones de los participantes, puede ser debido al desconocimiento que dichas entidades tienen de la metodología ApS, lo cual generó una disminución importante de la motivación de los alumnos. Esta falta de desarrollo íntegro de los proyectos ApS diseñados por los participantes no concuerda con la mayoría de las investigaciones relativas al ApS en la formación inicial de maestros, según las cuales los proyectos de ApS diseñados en este contexto se llevan a cabo con éxito en los lugares donde se ha detectado una necesidad social (Aramburuzabala y García, 2012; Blanch, París y Comes, 2017; Cuevas, Balaguer y Trigueros, 2017). Una posible causa que podría explicar esta diferencia, en relación con el grado de implementación, es que las necesidades sociales y los objetivos de servicio de los proyectos desarrollados en estas investigaciones están relacionados más directamente con la docencia, a diferencia de los analizados en este trabajo.

\section{CONCLUSIONES}

Los resultados de este trabajo revelan que los maestros en formación inicial participantes, de cuarto curso del Grado en Educación Primaria de la Universidad de Extremadura, son capaces de detectar las necesidades sociales de su entorno cercano (en concreto de su propio centro de estudios, de su lugar de reunión para hacer botellón, del río Guadiana y de la ciudad de Badajoz), como son la gestión de residuos (plásticos y papel) y el reciclaje, la falta de recursos básicos (comida y ropa) en grupos de población en riesgo de exclusión social o el consumismo existente en sus grupos sociales. Asimismo, estos alumnos son capaces de actuar sobre dichas necesidades sociales diseñando proyectos ApS con objetivos de servicio y de aprendizaje íntimamente relacionados, basados ambos en el conocimiento y la sensibilización acerca de las distintas necesidades sociales abordadas (reciclaje, especies invasoras, falta de recursos, consumismo...), las cuales son enmarcadas por los futuros maestros participantes como problemáticas a resolver para alcanzar los objetivos de desarrollo sostenible. Sin embargo, no logran trabajar el currículo de la asignatura en la profundidad que requiere la misma, aunque si plantean objetivos de aprendizaje relacionados con el temario de la misma.

Respecto a la difusión e implementación de los proyectos diseñados, los maestros en formación inicial participantes están capacitados para crear campañas de difusión y concienciación acerca de las necesidades sociales relacionadas con sus proyectos, proceso en el que otorgan un relevante papel a las redes sociales (Twitter, Facebook, Instagram...), desarrollando así el servicio a la comunidad que depende exclusivamente de ellos. Además, están capacitados para establecer contacto y proponer alianzas a diferentes entidades sociales, como son Cáritas, el comedor social de Badajoz, la Facultad de Educación y el Ayuntamiento de Badajoz. Las instituciones contactadas en la implementación de los proyectos desconocen qué es la metodología ApS y, en la mayor parte de los casos, solo son receptivas a colaborar en el proyecto en el papel de beneficiarias, rehusando implicarse en los objetivos de servicio, planteados en los proyectos ApS, que dependen exclusivamente de ellas. 


\section{Implicaciones en la formación inicial de maestros}

Los resultados de esta contribución, junto con los de investigaciones previas recogidas en el marco teórico, podrían tener algunas implicaciones en los procesos de formación inicial de maestros. Dado que el ApS es una metodología que favorece tanto el aprendizaje de contenidos concretos (de las asignaturas incluidas en los planes de estudios de los Grados en Educación Primaria) como de competencias relacionadas con la docencia, se sugiere incorporar el ApS como metodología de enseñanza-aprendizaje en alguna/s de las asignaturas de dichos Grados. De este modo, podría ser interesante formar a los futuros maestros en el ApS como metodología didáctica, así como mostrar diferentes ejemplos de proyectos ApS implementados con éxito para cubrir diferentes necesidades sociales de distintos entornos (rurales y urbanos, de clase socioeconómica baja y media...). Asimismo, en base a la experiencia desarrollada en este trabajo, podría ser muy valioso que sean los propios maestros en formación los que diseñen e implementen sus propios proyectos ApS en base a necesidades que ellos mismos detecten en su propio entorno. Sin embargo, de acuerdo con los resultados obtenidos en esta investigación, los objetivos de aprendizaje de estos proyectos ApS, planteados por los propios alumnos, tienen que ser redefinidos por el docente de la asignatura, para que alcancen el nivel exigido en la misma.

El diseño de proyectos ApS permitiría que los futuros docentes conozcan la metodología ApS y cómo implementarla (ventajas, potencialidades, dificultades...), a la vez que conozcan y reflexionen sobre las diferentes necesidades del entorno en el que ejercerán su futura acción docente. Así, a través de esta metodología, se podría contribuir a formar docentes más inclusivos, críticos e implicados en sus tareas docentes con su alumnado, pero también con la sociedad en general. Igualmente, teniendo en cuenta los problemas encontrados por los participantes en este trabajo a la hora de implementar sus proyectos ApS, consideramos necesario que las diferentes instituciones y entidades (educativas, gubernamentales, empresariales...) apoyen a los docentes, tanto en formación como en ejercicio, en las actividades desarrolladas dentro de estos proyectos. Este apoyo, como muestran los resultados de este trabajo, resulta clave para la consecución completa de los proyectos diseñados y, por tanto, para impulsar el ApS como metodología didáctica.

\section{Agradecimientos}

Este estudio ha sido financiado por el Proyecto EDU2016-77007-R (Ministerio de Ciencia e Innovación) y por la Ayuda a Grupos GR18004 (Junta de Extremadura)."

Elena Bravo Lucas agradece a la Junta de Extremadura por la concesión de un contrato FPI predoctoral (PD18045)".

\section{REFERENCIAS}

Álvarez, J. L., Martínez, M. J., González, H. y Buenestado, M. (2017). El aprendizaje-servicio en la formación del profesorado de las universidades españolas. Revista Española de Pedagogía, 75 (267), 199217. DOI: 10.22550/REP75-2-2017-02.

American Association of Higher Education (2002). Powerful partnerships: A shared responsibility for learning. In L. R. Lattuca, J. G. Haworth and C. F. Conrad (Eds.), College and University Curriculum: Developing and cultivating programs of study that enhance student learning (pp. 424-437). Boston: Pearson.

Anderson, J. (2000). Learning in deed: Service-learning and preservice teacher education. ECS Issue Paper. Education Commission of the States: Denver.

Aramburuzabala, P., García-Peinado, R. y Elvias, S. (2013). Educación desde y para la Justicia Social: una Experiencia de Aprendizaje-Servicio en la Formación de Maestros. En M.C. Pérez y M.M. Molero (Comps.), Variables psicólogicas y educativas para la intervención en el ámbito escolar (pp. 257265). Almería, España: Asociación Universitaria de Educación y Psicología.

Aramburuzabala, P., y García, R. (2012). El aprendizaje-servicio en la formación de maestros. Revista del Congrés Internacional de Docència Universitària i Innovació (CIDUI), 1(1). 
Ayuste, A, Gros, B., Payá, M., y Rubio, L. (2017). Valoración de proyectos ApS desde sus protagonistas: Un medio para la formación inicial de maestros/as. En J. Carrillo-Rosúa, J. L. Arco-Tirado y F. D. Fernández-Martín (Ed.), Investigando la mejora de la enseñanza universitaria a través del aprendizaje servicio/Researching the university teaching through service-learning (pp. 35-45). Granada, España: Editorial Universidad de Granada.

Blanch, S., París, G., y Comes, P. (2017). APS en los Prácticum del Grado de Educación y su evolución hacia el compromiso social. En J. Carrillo-Rosúa, J. L. Arco-Tirado y F. D. Fernández-Martín (Ed.), Investigando la mejora de la enseñanza universitaria a través del aprendizaje servicio/Researching the university teaching through service-learning (pp. 53-59). Granada, España: Editorial Universidad de Granada.

Briede, J. C., \& Mora, M. L. (2016). Diseño y co-creación mediante aprendizaje y servicio en contexto vulnerable: análisis de percepción de la experiencia. Formación universitaria, 9(1), 57-70.

Capella, C., Gil, J., Martí, M., y Chiva, Ó. (2015). Estudio de caso múltiple con historias de vida en el Grado de Educación Infantil: aprendizaje-servicio en la didáctica de la Educación Física. Profesorado. Revista de Currículum y Formación de Profesorado, 19(1), 334-348.

Coladarci, T. (1992). Teachers' Sense of Efficacy and Commitment to Teaching. Journal of Experimental Education, 60, 323-337.

Corbatón, R., Moliner, L., Martí, M., Gil, J., \& Chiva, Ó. (2015). Efectos académicos, culturales, participativos y de identidad del aprendizaje-servicio en futuros maestros a través de la educación física. Profesorado. Revista de Currículum y Formación de Profesorado, 19(1), 280-297.

CRUE (2015). Institucionalización del Aprendizaje-Servicio como estrategia docente dentro del marco de la Responsabilidad Social Universitaria para la promoción de la Sostenibilidad en la Universidad. Recuperado https://www.crue.org/Documentos\%20compartidos/Recomendaciones\%20y\%20criterios\%20tecnic os/2.\%20APROBADA\%20INSTITUCIONALIZACION\%20ApS.pdf

Cuevas, E., Balaguer, P., y Trigueros, C. (2017). Abriendo puertas hacia el aprendizaje servicio. Primer contacto con la metodología. En J. Carrillo-Rosúa, J. L. Arco-Tirado y F. D. Fernández-Martín (Ed.), Investigando la mejora de la enseñanza universitaria a través del aprendizaje servicio/Researching the university teaching through service-learning (pp. 385-390). Granada, España: Editorial Universidad de Granada.

Donahue, D. M. (1999). Service learning for preservice teachers: Ethical dilemmas for practice. Teaching and Teacher Education, 15, 685-695.

Folgueiras, P., Luna, E. y Puig, G. (2013). Aprendizaje y servicio: estudio del grado de satisfacción de estudiantes universitarios. Revista de Educación, 362, 159-185.

Francisco, A., \& Moliner, L. (2010). El Aprendizaje Servicio en la Universidad: una estrategia en la formación de ciudadanía crítica. Revista Electrónica Interuniversitaria De Formación del Profesorado, 13(4), 69-77.

García, M., \& Cotrina, M. J. (2015). El aprendizaje y servicio en la formación inicial del profesorado: de las prácticas educativas críticas a la institucionalización curricular. Profesorado: Revista de curriculum y formación del profesorado, 19(1), 8-25.

Gil, J. y Martí, M. (2012). Una experiencia intergeneracional: Innovación metodológica mediante la metodología del aprendizaje-servicio en educación infantil. Quaderns Digitals, 71.

Gillanders, C., Cores, A., \& Tojeiro, L. (2018). Educación musical y Aprendizaje-Servicio: estudio de caso en la formación de los futuros docentes de Educación Primaria. Revista Electrónica de LEEME, 2(42).

Ibarrola-García, S. y Artuch, R. (2016). La docencia en la universidad y el compromiso social y educativo. Contextos educativos, 19, 105-120.

Martín, M. Á., \& Rascón, D. (2015). La Educación Literaria: una oportunidad de aprendizaje servicio para la formación integral del futuro maestro. Profesorado. Revista de Currículum y Formación de Profesorado, 19(1), 350-366.

Martínez, M. (2008). Aprendizaje servicio y construcción de ciudadanía activa en la universidad: la dimensión social y cívica de los aprendizajes académicos. En M. Martínez. (Ed.), Aprendizaje servicio y responsabilidad social de las universidades (pp. 11-26). Barcelona, España: Ediciones Octaedro.

Opazo, H. (2015). Experiencias de aprendizaje-servicio en la formación del profesorado. Un estudio de caso (Tesis doctoral). Universidad Autónoma de Madrid, Madrid.

Ortega-Tudela, J. M., Cámara-Estrella, A. M., y Diaz-Pareja, E. M. (2015). Service learning as a tool to enhance future teachers' media competence / Aprendizaje-Servicio como estrategia favorecedora del desarrollo de la competencia mediática en futuros docentes. Cultura y Educación, 27(2), 440455.

Pérez, L. M., y Ochoa, A. C., (2017). El aprendizaje-servicio (APS) como estrategia para educar en ciudadanía. Alteridad. Revista de Educación, 12(2),175-187.

Priede, T., López-Cózar, C., Ruiz-Morales, C. A., y Baena, V. (2019). Descripción de una Experiencia de Aprendizaje basado en Retos para Contribuir a la Transformación Social. En F. J. Murillo y C. Martínez-Garrido (Coords.), Actas del XIX Congreso Internacional de Investigación Educativa Volumen V. Experiencias innovadoras y desarrollo socio-educativo (pp. 145-151). Madrid, España: Asociación Interuniversitaria de Investigación Pedagógica. 
Puig, J. M., y Palos, J. (2006). Rasgos pedagógicos del Aprendizaje-Servicio. Cuadernos de pedagogía, 357, 60-63.

Ramírez, C., Aramburuzabala, P. y Cerrillo, R. (2014). El proceso de institucionalización del AprendizajeServicio en la Facultad de Formación de Profesorado y Educación de la Universidad Autónoma de Madrid. En P. Aramburuzabala, H. Opazo y J. García-Gutiérrez (Eds.), El Aprendizaje-Servicio en las universidades. De la iniciativa individual a la institucional (pp.609-618). Madrid, España: UNED Ediciones.

Rodríguez, D., y Gómezescoba, A. (2019). Aprendizaje-Servicio (ApS) como estrategia de formación interdisciplinar de los futuros docentes de educación primaria. En M. López, A. M. Sanz y C. Pérez (Coords.), Experiencias de Innovación docente en Enseñanza Superior en Castilla-La Mancha 2019: III Jornada de Innovación Docente, Universidad de Castilla-La Mancha. (pp. 124-125). España: Ediciones de la Universidad de Castilla-La Mancha.

Rodríguez, P., de la Herrán, A., \& Cortina, M. (2015). Pedagogía de la muerte mediante aprendizaje servicio. Educación XX1, 18(1), 189-212.

Root, S. (2005). Improving Service-Learning Practice: Research on Models to Enhance Impact. En: S. Root, J. Callahan y S. Billig (Eds.), The National Service-Learning in Teacher Education Partnership: A Research Retrospective. Grenwich: Information Age.

Root, S., Callahan, J. y Sepanski, J. (2002). Service-learning in teacher education: A multisite study. Comunicación presentada en the Annual Meeting of the American Association of Colleges for Teacher Education, New York.

Santos-Pastor, M. L., Cañadas, L., y Martínez-Muñoz, L. F. (2020). Limitaciones del aprendizaje-servicio en la formación inicial en actividad físico-deportiva / Limitations of service-learning in initial training in physical activity and sports. Retos, 37(37), 509-517.

Tapia, M. (2010). La propuesta pedagógica del "aprendizaje-servicio": una perspectiva Latinoamericana. Revista científica TzhoeCoen, 3(5), 23-44.

Tello, I., Cerrillo, R., Mateos, S., y Aramburuzabala, P. (2017). Mejora de la formación inicial de docentes de infantil y primaria mediante la implementación de la metodología de aprendizaje-servicio. En F. Murillo (Coord.), Avances en liderazgo y mejora de la educación: Actas del I Congreso Internacional de Liderazgo y Mejora de la Educación. (pp. 189-194). Madrid, España: RILME.

Vázquez, S., Liesa, M. \& Lozano, A. (2017). Recreos Cooperativos e Inclusivos a través de la metodología de Aprendizaje-Servicio. Revista electrónica interuniversitaria de formación del profesorado, 20(1), 173-185.

Wee, C. (2017). La opinión de los estudiantes sobre el aprendizaje-servicio y sus efectos en la formación docente. Debates \& Prácticas en Educación, (2), 36-53. 